\title{
Relationship between LET and RBE values for Escherichia coli determined using carbon ion beams from the TIARA cyclotron and HIMAC synchrotron
}

\author{
Masahiro Imamura, Takashi Murata, Kiyoshi Akagi, Yoshimasa Tanaka, Masato Imamura, ${ }^{1}$ Kyoichi Inoue, ${ }^{1}$ \\ Nagayo Mizuma, ${ }^{2}$ Yasuhiko Kobayashi, ${ }^{3}$ Hiroshi Watanabe,${ }^{3}$ Misao Hachiya, ${ }^{4}$ Makoto Akashi, ${ }^{4}$ \\ Yoshiya Furusawa, ${ }^{5}$ Hisashi Yamanaka, ${ }^{6}$ Shinpei Takahashi, ${ }^{7}$ Tamotsu Nakano, ${ }^{7}$ \\ Shunji Nagaoka, ${ }^{7}$ Takeo Ohnishi, ${ }^{8}$ Yurino Obiya, ${ }^{9}$ and Kazuki Harada ${ }^{9, *}$ \\ Department of Radiology, and ${ }^{1}$ Third Department of Internal Medicine, Kansai Medical University, Moriguchi 570, Japan \\ ${ }^{2}$ Research Reactor Institute, Kyoto University, Sennan-gun, Osaka 590-04, Japan \\ ${ }^{3}$ Takasaki Radiation Chemistry Research Establishment, Japan Atomic Energy Research Institute (JAERI), Takasaki 370-12, Japan \\ ${ }^{4}$ Division of Radiation Health, and ${ }^{5}$ Space and Particle Radiation Science Research Group, \\ National Institute of Radiological Science (NIRS), Chiba 263, Japan \\ ${ }^{6}$ Research and Survey Department, Japan Space Utilization Promotion Center (JSUP), Shinjuku-ku, Tokyo 169, Japan \\ ${ }^{7}$ Space Experiment Group, National Space Development Agency of Japan (NASDA), Tsukuba 305, Japan \\ ${ }^{8}$ Department of Biology, Nara Medical University, Kashihara 634, Japan \\ ${ }^{9}$ Division of Molecular Life Science, PL Botanical Institute, PL Gakuen Women's Junior College, Tondabayashi 584, Japan
}

(Received March 7, 1997; Accepted April 21, 1997)

Key Words_carbon ion beam; DNA repair; Escherichia coli; HIMAC; KURRI; LET; RBE; TIARA

Humans have to overcome two obstacles for extended periods of manned space exploration: microgravity in the space environment and the presence of high-energy cosmic heavy-particle radiation in space (Malacinski and Neff, 1990). Since there is little knowledge of the effects of cosmic heavy-particle radiation on living organisms in space, studies of this aspect are very important.

In the future, it is hoped that radioprotective systems for humans in space will be developed through space radiation biology research. Such investigations must examine the effects of cosmic heavy-particle radiation on living organisms and the mechanisms involved. As only a few experiments have been conducted in space, simulation experiments conducted on earth are necessary, and this can be accomplished by utilizing cyclotrons and synchrotrons which generate heavy-particle radiation (heavy-ion beams) (Harada et al., 1992).

In this study, we investigated the cell lethality of ion beams, some of the cosmic heavy ions originating

\footnotetext{
* Address reprint requests to: Dr. Kazuki Harada, Division of Molecular Life Science, PL Botanical Institute, PL Gakuen Women's Junior College, 1 Kamiyama-cho, Tondabayashi 584, Japan.
}

from galaxies, using two strains of the prokaryote Escherichia coli: the wild-type strain and a DNA repairdeficient mutant strain lacking the ability to repair DNA damage. Furthermore, we studied the relationship between the linear energy transfer (LET) and relative biological effectiveness (RBE) values for $E$. coli cell lethality from the data of $D_{10}$ values as the dose quantity necessary for decreasing the survival fraction to $10 \%$.

E. coli strains KMBL3835 (trpE9777- ${ }^{-}$, wild-type with respect to DNA repair activity) and KY385 (trpE9777-, recA56 ${ }^{-}$) were used. The ultraviolet (UV) light sensitivity of these strains has already been reported (Obiya and Harada, 1994), and it is known that sensitivity increases in the order of KMBL3835, KY386 (trpE9777 ${ }^{-}$, uvrA6 ${ }^{-}$) and $\mathrm{KY} 385$. For UV irradiation, the $D_{10}$ of strain $K Y 385$ is about $2 \mathrm{~J} / \mathrm{m}^{2}$.

The KMBL3835 and KY385 strains were exposed to low-LET $\gamma$ radiation at the ${ }^{60} \mathrm{Co}$ irradiation facilities of the Research Reactor Institute, Kyoto University (KURRI), and the results were compared with those obtained using high-LET radiation in the form of boron neutron captured (BNC) beams (mainly $\alpha$-particles) from the nuclear reactor at the same institute. For exposure to $\alpha$-particles, the cells were suspended in 
saline containing $0.3 \mathrm{M}{ }^{10} \mathrm{~B}$-enriched boric acid $\left(\mathrm{H}_{3}{ }^{10} \mathrm{BO}_{3} ; 90.2 \%\right)$. The $\alpha$-particles were generated by the nuclear reaction ${ }^{10} \mathrm{~B}(n, \alpha)^{7} \mathrm{Li}$ from thermal neutrons $\left(6 \times 10^{9} \mathrm{n} / \mathrm{cm}^{2} \mathrm{~s}\right)$ in the heavy-water $\left(D_{2} \mathrm{O}\right)$ facility of the reactor operated at $5 \mathrm{MW}$. The LET value of the $\alpha$-particles was about $230 \mathrm{keV} / \mu \mathrm{m}$ (Kobayashi and Kanda, 1982). Similarly, these strains were exposed to another source of high-LET radiation in the form of carbon ions. The ${ }^{12} \mathrm{C}^{5+}$ ion radiation was generated from an AVF cyclotron at an energy of $220 \mathrm{MeV}$ and LET of $121 \mathrm{keV} / \mu \mathrm{m}$ at the Takasaki Ion Accelerator for Radiation Application (TIARA) facility of the Japanese Atomic Energy Institute (JAERI) in Takasaki, Japan. The ${ }^{12} \mathrm{C}^{6+}$ ion radiation (mono-beam) was generated from the synchrotron at an energy of $290 \mathrm{MeV} / \mathrm{u}$ and LET of $80 \mathrm{keV} / \mu \mathrm{m}$ at the Heavy Ion Medical Accelerator in Chiba (HIMAC) facility at the National Institute of Radiological Science (NIRS) in Chiba, Japan. For all

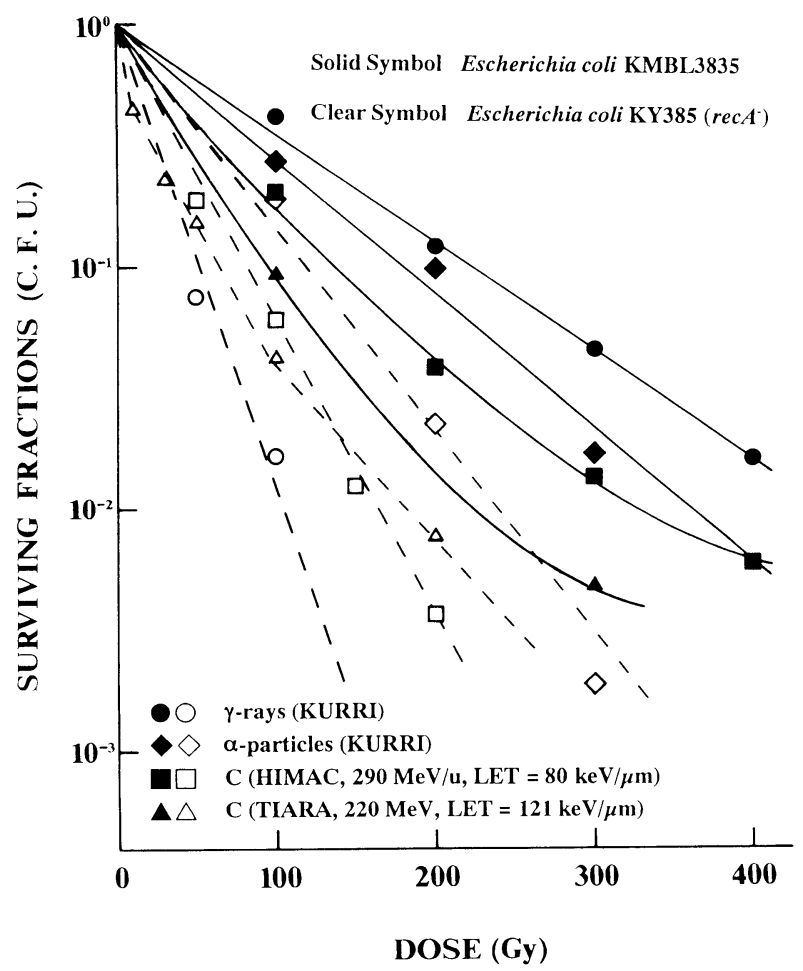

Fig. 1. Dose-response survival curves of Escherichia coli KMBL3835 (trpE9777 ${ }^{-}$) and KY385 (trpE9777 ${ }^{-}$, recA56 ${ }^{-}$) cells irradiated with $\alpha$-particles, $C$ ions and $\gamma$-rays.

The cells were grown at $37^{\circ} \mathrm{C}$ in a broth medium (Ohnishi et al., 1983) containing $1.0 \%$ Bacto beef extract (Difco, Detroit, MI, U.S.A.), $1.0 \%$ polypepton and $0.2 \% \mathrm{NaCl}$, adjusted to $\mathrm{pH} 7.4$. Growth was followed by measuring the optical density at $550 \mathrm{~nm}$ using a recording spectrophotometer (Shimadzu UV-240; Shimadzu, Kyoto, Japan) (Obiya and Harada, 1994). Cell growth was stopped at the late logarithmic phase. After irradiation, the diluted samples were spread on broth agar plates made by solidifying the broth medium with $2.0 \%$ Bacto-agar (Difco). Colonies for measurement of survival were counted after incubation at $37^{\circ} \mathrm{C}$ for $24 \mathrm{~h}$. Each survival point was the mean of six plates with cells diluted at three different steps. exposures to radiation, cells suspended in saline were used. After irradiation, their survival fractions were compared.

The results for the surviving fractions are shown in Fig. 1. Strain KMBL3835 showed a higher tolerance than KY385 to all four sources of radiation, and the sensitivity of the wild-type strain increased in the order of $\gamma$-rays, $\alpha$-particles, ${ }^{12} \mathrm{C}^{6+}$ and ${ }^{12} \mathrm{C}^{5+}$. This difference seemed to be attributable to the difference in LET; the sensitivity appeared to increase with increasing LET up to $121 \mathrm{keV} / \mu \mathrm{m}$.

Each $D_{10}$ value was determined from the survival curves in Fig. 1. These values were: $D_{10} \gamma$-rays (KMBL3835) $223 \mathrm{~Gy} ; D_{10 \gamma \text {-rays (KY385) }} 50 \mathrm{~Gy}$; $D_{10} \alpha$-particles (KMBL3835) 180 Gy; $D_{10 \alpha \text {-particles (KY385) }} 118 \mathrm{~Gy} ; \mathrm{D}_{10}{ }^{12} \mathrm{C}^{6+}$ (KMBL3835) $138 \mathrm{~Gy}$; $\mathrm{D}_{10}{ }^{12} \mathrm{C}^{6+}$ (KY385) $83 \mathrm{~Gy} ; \mathrm{D}_{10}{ }^{12} \mathrm{C}^{5+}$ (KMBL3835) $100 \mathrm{~Gy}$; and $D_{10}{ }^{12} C^{5+}(K Y 385) 63$ Gy. The LET value was already determined from the character of the ion and the machine capability. The RBE value was calculated as the ratio of $D_{10 \gamma \text {-ray }}$ value/ $D_{10}$ ion beam value. We then plotted the relationship between LET (horizontal line) and RBE (vertical line) for cell lethality on $E$. coli KMBL3835 wild-type strain (Fig. 2).

It has been generally believed that prokaryotic cells do not have a RBE peak in plots of LET against RBE for cell lethality, whereas eukaryotic cells have a peak at about $100 \mathrm{keV} / \mu \mathrm{m}$ LET (Kondo, 1972). In this experiment, we found for the first time that a prokaryote, the E. coli wild-type strain, has a RBE peak at $121 \mathrm{keV} / \mu \mathrm{m}$; the RBE value being 1.81. However, this was not the case for the mutant strain.

Furthermore, we found that the ratio of the $D_{10}$ value for strain KMBL3835 to that for strain KY385 (i.e., the $D_{10}$ ratio $\left(D_{10} \mathrm{kMBL} 3835\right.$ value/ $D_{10} \mathrm{kY} 385$ value) observed after $\gamma$ irradiation) was larger than that after irradiation with $\alpha$-particles and carbon ions. This indicated that the sensitivity of strain $\mathrm{KY} 385$, which lacks recombination repair ability, increases more slowly than that of the KMBL3835 wild-type strain as the LET value in-

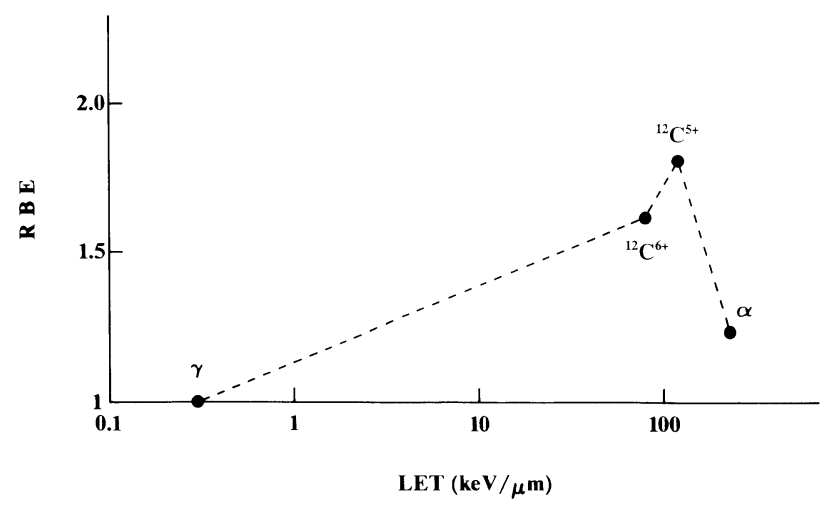

Fig. 2. The relationship between linear energy transfer (LET) and relative biological effectiveness (RBE) for Escherichia coli strain KMBL3835. 
creases. Therefore, whereas the $\operatorname{rec} A$ gene greatly contributes to the repair of DNA damage caused by $\gamma$ irradiation $\left(D_{10}\right.$ ratio $\left.=4.46\right)$, the contribution of this gene for the repair of DNA damaged by $\alpha$-particles $\left(D_{10}\right.$ ratio $\left.=1.53\right)$ and carbon ions $\left(D_{10}{ }^{12} \mathrm{C}^{6+}\right.$ ratio $=1.66$ or $\mathrm{D}_{10}{ }^{12} \mathrm{C}^{5+}$ ratio $=1.59$ ) seems to become smaller according to the increase of LET value; suggesting increased cell lethality.

We have already observed the presence of hot spots (specific areas) for mutation on DNA molecules of the shuttle vector plasmid pZ189 using a BNC beam containing $\alpha$-particles and the DNA base sequence method (Nakano et al., 1995). These two strains of $E$. coli are auxotrophic mutants, so that back-mutation can be examined by observing their growth on minimal medium (Ohnishi et al., 1980). Furthermore, the lethality and mutagenicity of various heavy ions on $E$. coli DNA repair-deficient mutants will be investigated.

The authors are grateful to Mr. Tatsuo Nakano (PL Botanical Institute, PL Gakuen Women's Junior College) for technical support. This work was carried out in part under the Visiting Researcher's Program of the Research Reactor Institute, Kyoto University, the TIARA Cooperative Research Project on Ion Beams at JAERI and the Research Project with Heavy lons at NIRS-HIMAC. This work was also carried out in part as a joint research program with the Japan Space Utilization Promotion Center. The study was supported in part by a Grant-in-Aid from the Japan Private School Promotion Foundation (Special Educational Research Project) and by
The Katano Grant awarded by the Alumni Association of Kansai Medical University.

\section{References}

Harada, K., Kimura, R., Oda, S., Kawashima, M., Miki, T., Mizuma, N., Maki, H., Saito, M., Watanabe, H., and Tano, S. (1992) Synergistic killing effect of alpha particle beam and hyperthermia on Deinococcus radiodurans. In Proceedings of International Conference on Evolution in Beam Applications, ed. by JAERI, Radiation Application Development Association, Ibaraki/Gunma, pp. 593-598.

Kobayashi, T. and Kanda, K. (1982) Analytical calculation of boron10 dosage in cell nucleus for neutron capture therapy. Radiat. Res., 91, 77-94.

Kondo, S. (1972) Molecular Radiation Biology (in Japanese), Japan Scientific Societies Press, Tokyo, pp. 173-176.

Malacinski, G. M. and Neff, A. W. (1990) Introduction to space biology. In Fundamentals of Space Biology, ed. by Asashima, M. and Malacinski, G., Japan Scientific Societies Press/SpringerVerlag, Tokyo/Berlin, pp. 1-7.

Nakano, T., Okaichi, K., Harada, K., Matsumoto, H., Kimura, R., Yamamoto, K., Akasaka, S., and Ohnishi, T. (1995) Mutations of a shuttle vector plasmid, pZ189, in Escherichia coli induced by boron neutron captured beam (BNCB) containing $\alpha$-particles. Mutat. Res., DNA Repair, 336, 153-159.

Obiya, Y. and Harada, K. (1994) Preparation of Escherichia coli for Space Shuttle experiment "IML-2 project." PL G. W. J. C. Bull., 21, 1-7.

Ohnishi, T., Ohashi, Y., Nozu, K., and Inoki, S. (1980) Mutagenicity of nifurtimox in Escherichia coli. Mutat. Res., 77, 241-244.

Ohnishi, T., Ohashi, Y., Nozu, K., and Inoki, S. (1983) Mutagenicity of anti-trypanosomal drug, Ro7-1051 in Escherichia coli. Jpn. J. Genet., 58, 505-509. 